\title{
Opposing Effects of Aging on Large-Scale Brain Systems for Memory Encoding and Cognitive Control
}

\author{
Alireza Salami, ${ }^{1,2}$ Johan Eriksson, ${ }^{1,2}$ and Lars Nyberg ${ }^{1,2,3}$ \\ ${ }^{1}$ Umeå Center for Functional Brain Imaging, S-90187, Umeå, Sweden, ${ }^{2}$ Department of Integrative Medical Biology, Physiology Section, and ${ }^{3}$ Department of \\ Radiation Sciences, Diagnostic Radiology, Umeå University, S-901 87 Umeå, Sweden
}

Episodic memory declines with advancing age. Neuroimaging studies have associated such decline to age-related changes in general cognitive-control networks as well as to changes in process-specific encoding or retrieval networks. To assess the specific influence of aging on encoding and retrieval processes and associated brain systems, it is vital to dissociate encoding and retrieval from each other and from shared cognitive-control processes. We used multivariate partial-least-squares to analyze functional magnetic resonance imaging data from a large population-based sample $(n=292,25-80$ years $)$. The participants performed a face-name paired-associates task and an active baseline task. The analysis revealed two significant network patterns. The first reflected a process-general encoding-retrieval network that included frontoparietal cortices and posterior hippocampus. The second pattern dissociated encoding and retrieval networks. The anterior hippocampus was differentially engaged during encoding. Brain scores, representing whole-brain integrated measures of how strongly an individual recruited a brain network, were correlated with cognitive performance and chronological age. The scores from the general cognitive-control network correlated negatively with episodic memory performance and positively with age. The encoding brain scores, which strongly reflected hippocampal functioning, correlated positively with episodic memory performance and negatively with age. Univariate analyses confirmed that bilateral hippocampus showed the most pronounced activity reduction in older age, and brain structure analyses found that the activity reduction partly related to hippocampus atrophy. Collectively, these findings suggest that age-related structural brain changes underlie age-related reductions in the efficient recruitment of a process-specific encoding network, which cascades into upregulated recruitment of a general cognitive-control network.

\section{Introduction}

Episodic memory (Tulving, 1983) is the most age-sensitive longterm memory system (Nyberg et al., 1996b; Rönnlund et al., 2005). Imaging studies have linked episodic memory impairment in aging to several key regions of a large-scale episodic memory network, including medial temporal lobe (MTL; Grady et al., 1995), parietal (Nyberg et al., 2003), and frontal (Cabeza et al., 1997) regions. However, the process-impure nature of cognitive tasks (Foster and Jelicic, 1999) makes it difficult to say whether observations in imaging studies of age-related changes in functional networks is indicative of a core episodic memory deficit or whether it reflects more general working-memory and/or executive deficits (Gazzaley et al., 2008).

Moreover, it remains unclear whether aging selectively impairs encoding or retrieval processes, or whether it has more

\footnotetext{
Received Jan. 19, 2012; revised May 4, 2012; accepted June 14, 2012.

Author contributions: L.N. designed research; A.S., J.E., and L.N. performed research; A.S. analyzed data; A.S. and L.N. wrote the paper.

This study was supported by the Göran Gustafsson Award in Medicine (L.N.), a grant from the Swedish Science Council (L.N.), and a Wallenberg Scholar Grant from the Knut and Alice Wallenberg Foundation (L.N.). The BETULA Project is supported by a grant from the Swedish Science Council (L.-G. Nilsson and L.N.). We thank the staff of the BETULA Project, R. Adolfsson and L.-G. Nilsson, and the staff at the Umeå Center for Functional Brain Imaging (especially Micael Andersson).

Correspondence should be addressed to Alireza Salami, Department of Integrative Medical Biology, Umeå Center for Functional Brain Imaging, Umeå University, S-901 87 Umeå, Sweden. E-mail: alireza.salami@physiol.umu.se. DOI:10.1523/JNEUROSCI.0278-12.2012

Copyright $\odot 2012$ the authors $\quad 0270-6474 / 12 / 3210749-09 \$ 15.00 / 0$
}

process-general mnemonic influences. Some studies have found that certain MTL regions are jointly activated during encoding and retrieval (Nyberg et al., 1996c; Greicius et al., 2003), but there is also evidence for functional specialization (Gabrieli, 1997; Rombouts et al., 2001; Giovanello et al., 2009). Similarly, there is evidence that various episodic encoding and retrieval conditions engage overlapping frontal regions (Nyberg et al., 1996c), but also that specific frontal regions are differentially engaged during encoding versus retrieval (Cabeza and Nyberg, 2000).

To specifically assess the influence of aging on episodic memory encoding and retrieval processes and associated brain systems, it is vital to dissociate encoding and retrieval from each other as well as from shared cognitive-control processes. In the present study, we used a multivariate partial-least-squares (PLS) analysis method (McIntosh and Lobaugh, 2004) to approach this issue. The PLS method has previously been used to identify common as well as process-specific patterns of brain regions involved in episodic encoding and retrieval (Nyberg et al., 1996c). PLS accommodates indices of both similarities and differences by considering all experimental variables, simultaneously. Thus, if encoding and retrieval engage many of the same brain regions, PLS should reveal a common network. Alternatively, or in addition, if some brain regions are differentially engaged during encoding and retrieval, PLS can reveal process-specific encoding and retrieval networks.

PLS was applied to data from a large-scale sample $[N=292$, 25-80 years of age (Salami et al., 2012)] from a population-based 


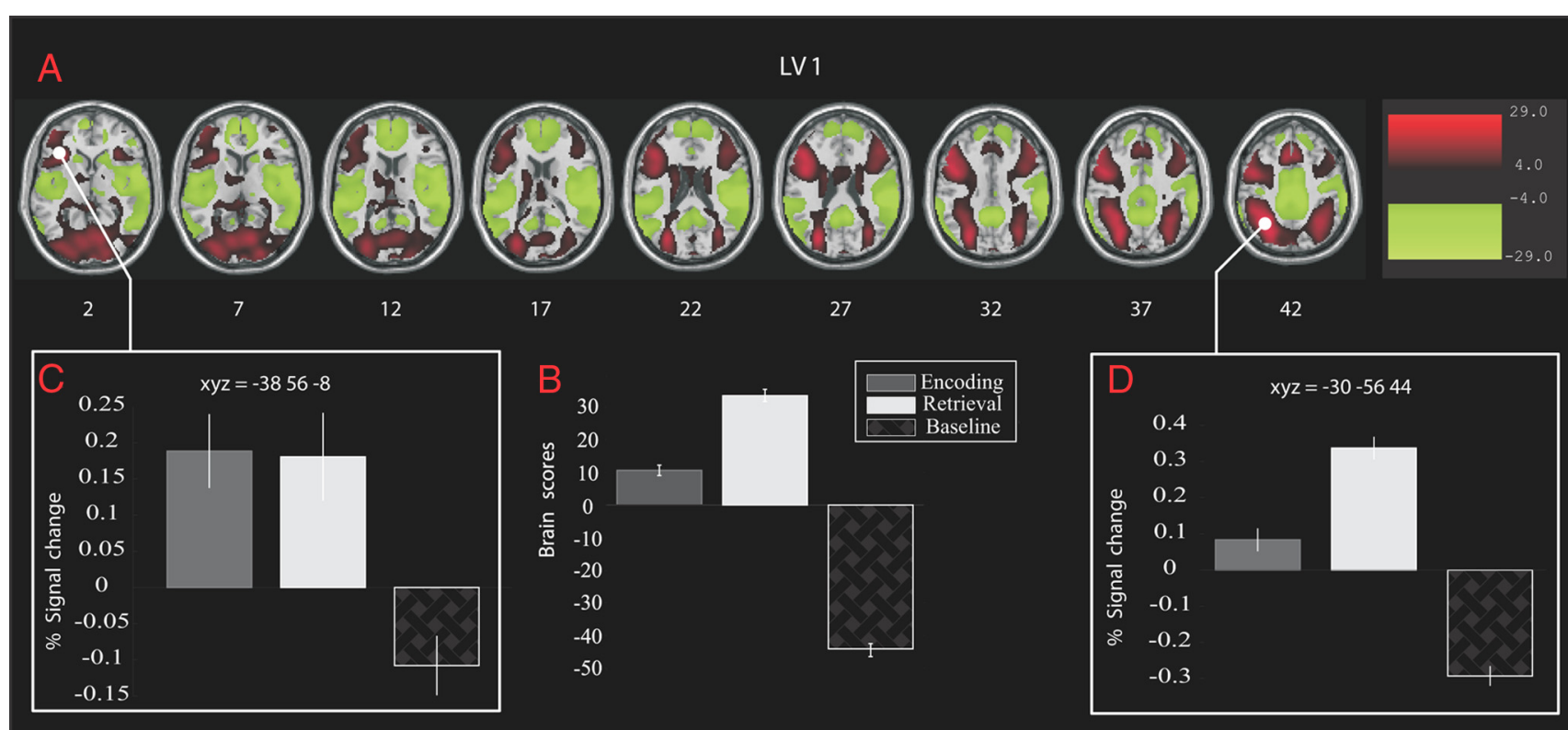


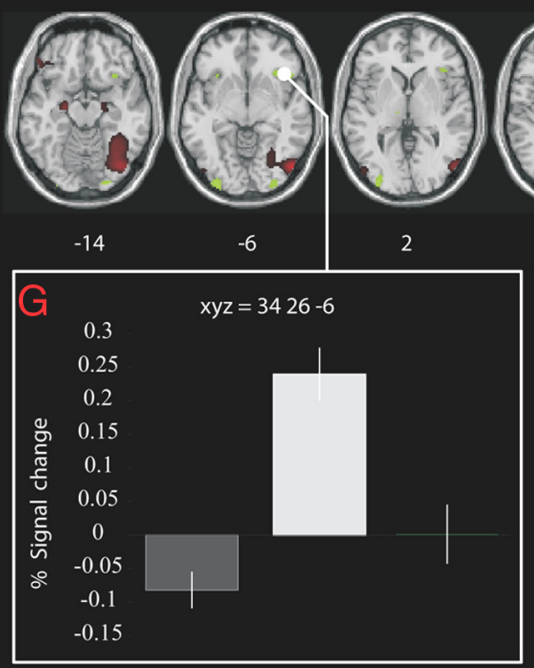

LV2

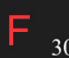

F 30

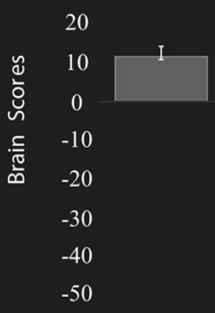

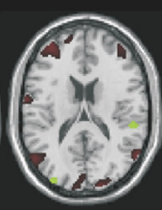

18

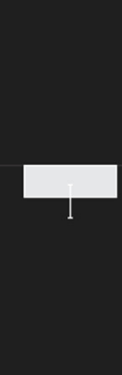

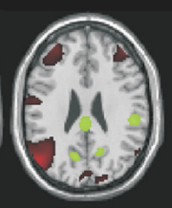
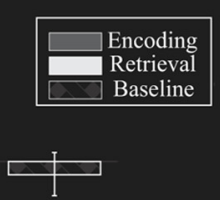
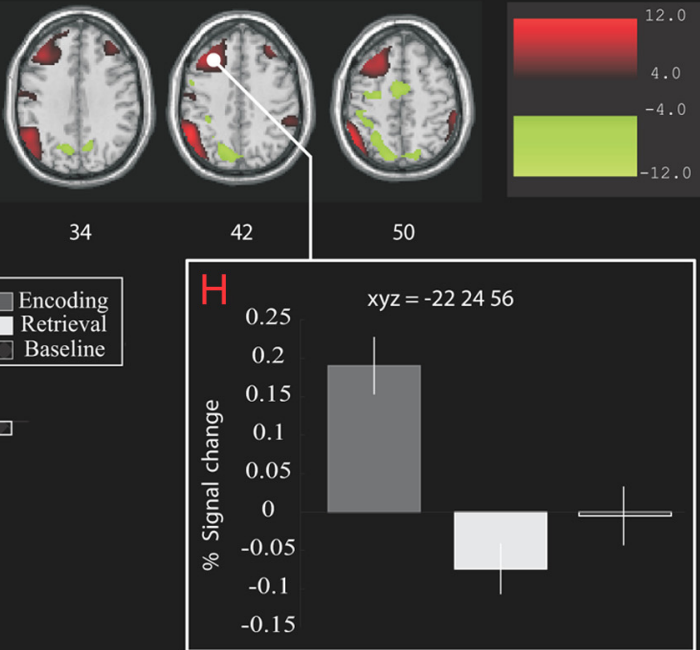

Figure 1. Singular images, brain scores, and signal changes (for selected local maxima) for the two significant LVs. $\boldsymbol{A}, \boldsymbol{E}$, Singular images for LV1 and LV2, which display regions reliably contributing to the pattern identified in each LV. For LV1, red represents regions showing greater activity during encoding and retrieval relative to baseline, whereas green represents brain regions more active during baseline than during encoding and retrieval. $B, F$, Brain scores for LV1 and LV2, reflecting commonality and differentiation, respectively, for episodic encoding and retrieval. $C, D$, Signal changes in two regions contributing to LV1. Error bars indicate 1 SE. G, $\boldsymbol{H}$, Signal changes in left and right PFC, highlighting the HERA model.

study [Betula (Nilsson et al., 1997, 2004; Nyberg et al., 2010)]. The participants were scanned with functional magnetic resonance imaging ( $\mathrm{fMRI}$ ) while they engaged in a face-name pairedassociates (FN-PA) encoding and retrieval task (Sperling et al., 2001; Zeineh et al., 2003) as well as an active baseline task (Stark and Squire, 2001). They also took part in a detailed cognitive test session that included tests of episodic memory, semantic memory, and visuospatial ability. We correlated individual "brain scores" from the PLS analysis, representing an integrated measure of how strongly an individual recruited a certain brain network, with memory performance and chronological age (Grady et al., 2006). We expected process-specific episodic networks to be positively related to performance on episodic tasks (but not other cognitive tasks) and negatively related to chronological age.

\section{Materials and Methods}

Participants. All participants in the present analyses $(N=292,152 \mathrm{fe}-$ males) were part of the Betula Prospective Cohort Study on memory, health, and aging. Participants' ages ranged from 25 to 80 years (mean $=$ $59.5 \pm 13.1$ years). From the initial sample of 376 participants, 84 were excluded. Reasons for exclusion included low task performance (lower than chance level; 62 participants, $73.8 \%$ ), not performing the task at all (9 participants, $10.7 \%$ ), technical error in E-prime (1 participant, 1.1\%), misunderstanding the task ( 1 participant, $1.1 \%$ ), pathology ( 1 participant, 1.1\%), and corrupted T1-weighted structural image (10 participants, $11.9 \%$ ). All remaining participants were Swedish native speakers, had normal or corrected to normal vision, and had no history of severe neurological illness or events that might cause dementia. The older adults were examined by a physician and were generally healthy with no evidence of severe health problems (e.g., heart or kidney diseases). Informed 
Table 1. Regions from LV1 showing general process network of episodic memory

\begin{tabular}{|c|c|c|}
\hline Regions & $X Y Z$ & BSR \\
\hline \multicolumn{3}{|l|}{ Positive direction } \\
\hline R-fusiform & {$[24-82-16]$} & 44.84 \\
\hline L-mid-occipital & {$\left[\begin{array}{lll}-32 & -886\end{array}\right]$} & 44.15 \\
\hline L-fusiform & {$\left[\begin{array}{llll}-40 & -70 & -14\end{array}\right]$} & 43.57 \\
\hline L-inf-parietal & {$\left[\begin{array}{lll}-30 & -56 & 44\end{array}\right]$} & 42.63 \\
\hline L-insula & {$\left[\begin{array}{lll}-32 & 22 & -2\end{array}\right]$} & 40.30 \\
\hline R-cerebellum & {$[34-56-20]$} & 38.78 \\
\hline R-insula & {$\left[\begin{array}{lll}32 & 24 & -2\end{array}\right]$} & 36.48 \\
\hline L-inf-frontal & {$\left[\begin{array}{llll}-44 & 22 & 24\end{array}\right]$} & 35.76 \\
\hline R-mid-occipital & {$\left[\begin{array}{lll}32 & -70 & 28\end{array}\right]$} & 35.12 \\
\hline L-cerebellum & {$\left[\begin{array}{lll}-28 & -56 & -20\end{array}\right]$} & 34.48 \\
\hline L-mid-frontal & {$[-26-254]$} & 30.42 \\
\hline R-sup-parietal & {$[22-7050]$} & 29.83 \\
\hline R-lingual & {$[8-70-6]$} & 29.77 \\
\hline R-mid-frontal & {$[34460]$} & 28.11 \\
\hline R-inf-frontal & {$\left[\begin{array}{llll}46 & 18 & 30\end{array}\right]$} & 26.47 \\
\hline L-hippocampus & {$[-30-342]$} & 24.52 \\
\hline R-hippocampus & {$\left[\begin{array}{lll}38 & -32 & -4\end{array}\right]$} & 21.27 \\
\hline L-mid-frontal & {$\left[\begin{array}{lll}-38 & 56 & -8\end{array}\right]$} & 20.57 \\
\hline R-precuneus & {$[4-7258]$} & 18.81 \\
\hline L-precuneus & {$\left[\begin{array}{lll}-4 & -50 & 72\end{array}\right]$} & 15.84 \\
\hline R-sup-frontal & {$\left[\begin{array}{lll}28 & 60 & -4\end{array}\right]$} & 12.46 \\
\hline R-inf-frontal & {$\left[\begin{array}{llll}50 & 44 & -12\end{array}\right]$} & 8.68 \\
\hline L-paracentral & {$[-2-1474]$} & 4.53 \\
\hline \multicolumn{3}{|l|}{ Negative direction } \\
\hline R-supramarginal & {$\left[\begin{array}{lll}60 & -26 & 24\end{array}\right]$} & 25.20 \\
\hline R-mid-cingulum & {$[10-2844]$} & 24.09 \\
\hline R-insula & {$[40-12-2]$} & 23.90 \\
\hline L-sup-temporal & {$[-40-6-12]$} & 23.65 \\
\hline L-mid-cingulum & {$\left[\begin{array}{lll}-10 & -30 & 42\end{array}\right]$} & 19.54 \\
\hline R-sup-frontal & {$\left[\begin{array}{lll}6 & 56 & 20\end{array}\right]$} & 15.89 \\
\hline L-caudate & {$\left[\begin{array}{lll}-16 & 22 & -2\end{array}\right]$} & 11.78 \\
\hline R-sup-temporal & {$\left[\begin{array}{lll}64 & -56 & 18\end{array}\right]$} & 11.72 \\
\hline R-caudate & {$\left[\begin{array}{lll}16 & 24 & -2\end{array}\right]$} & 11.61 \\
\hline R-mid-temporal & {$\left[\begin{array}{lll}64 & -58 & 6\end{array}\right]$} & 11.19 \\
\hline L-sup-frontal & {$\left[\begin{array}{llll}-12 & 46 & 40\end{array}\right]$} & 10.34 \\
\hline L-putamen & {$\left[\begin{array}{lll}-12 & 12 & 6\end{array}\right]$} & 9.76 \\
\hline L-mid-frontal & {$\left[\begin{array}{lll}-28 & 26 & 42\end{array}\right]$} & 6.87 \\
\hline
\end{tabular}

BSR, bootstrap ratio; $B S R>4, p<0.0001$.

consent was signed by all participants, in accordance with guidelines of the Swedish Council for Research in the Humanities and Social Sciences.

fMRI activation task. The FN-PA task was used, which consists of 20 blocks (in total) of encoding, retrieval, and control tasks, alternating during the scanning session. Within each encoding and retrieval block (six blocks each), four stimuli were presented for $4 \mathrm{~s}$ each, with a randomized interstimulus interval of $1.5,2.5,3$, or 4.5 s. Block-specific instructions were provided to participants before each block for $4 \mathrm{~s}$. During the encoding blocks, the face-name stimuli were presented and participants were instructed to remember the name associated with each face. They were also instructed to press a button, using the index finger, to indicate that they saw a face-name pair. The faces (an equal number of males and females) were digital color photographs portraying persons, unknown to the participants. The names were fictional first names, printed in white on a black background, that were paired with the faces by the investigators.

During the retrieval blocks, each face was presented along with three letters of which one corresponded to the first letter of the previously encoded face-name pair. The task was to indicate the letter corresponding to the name that was previously encoded with the face. The top, middle, and bottom letters corresponded to the index, the ring, and the middle fingers, respectively. Participants were instructed to respond by guessing if they could not remember the face-name pair.

An active baseline task was used to dissociate different aspects of episodic memory from sensory motor components. Also, it has been shown that activity in the MTL is substantially higher during rest than during
Table 2. Regions from LV2 reflecting process-specific networks of episodic memory

\begin{tabular}{|c|c|c|}
\hline Regions & $X Y Z$ & BSR \\
\hline \multicolumn{3}{|l|}{ Positive direction } \\
\hline L-lingual & {$\left[\begin{array}{lll}-16 & -92 & -12\end{array}\right]$} & 19.13 \\
\hline L-hippocampus & {$[-28-14-16]$} & 16.12 \\
\hline L-sup-frontal & {$\left[\begin{array}{llll}-22 & 24 & 56\end{array}\right]$} & 14.32 \\
\hline L-mid-frontal & {$\left[\begin{array}{llll}-34 & 20 & 54\end{array}\right]$} & 14.24 \\
\hline L-postcentral & {$\left[\begin{array}{llll}-48 & -14 & 38\end{array}\right]$} & 11.85 \\
\hline R-hippocampus & {$[24-8-18]$} & 10.35 \\
\hline R-postcentral & {$[58-226]$} & 8.09 \\
\hline R-mid-temporal & {$\left[\begin{array}{lll}60 & -60 & 20\end{array}\right]$} & 7.68 \\
\hline L-inf-frontal & {$\left[\begin{array}{lll}-38 & 34 & -14\end{array}\right]$} & 7.01 \\
\hline R-mid-frontal & {$\left[\begin{array}{lll}36 & 36 & 44\end{array}\right]$} & 6.47 \\
\hline L-mid-frontal & {$\left[\begin{array}{llll}-34 & 50 & 22\end{array}\right]$} & 6.31 \\
\hline L-inf-frontal & {$\left[\begin{array}{lll}-40 & 52 & -10\end{array}\right]$} & 4.91 \\
\hline \multicolumn{3}{|l|}{ Negative direction } \\
\hline L-postcentral & {$\left[\begin{array}{lll}-38 & -34 & 44\end{array}\right]$} & 20.06 \\
\hline R-inf-frontal & {$\left[\begin{array}{lll}34 & 26 & -6\end{array}\right]$} & 17.72 \\
\hline L-insula & {$\left[\begin{array}{lll}-30 & 25 & -4\end{array}\right]$} & 17.32 \\
\hline L-mid-cingulum & {$[-4-450]$} & 14.15 \\
\hline L-sup-parietal & {$\left[\begin{array}{lll}-28 & -54 & 66\end{array}\right]$} & 13.33 \\
\hline R-cerebellum & {$\left[\begin{array}{lll}22 & -52 & -26\end{array}\right]$} & 12.15 \\
\hline R-mid-cingulum & {$\left[\begin{array}{lll}8 & 20 & 34\end{array}\right]$} & 11.01 \\
\hline R-sup-temporal & {$\left[\begin{array}{lll}60 & -30 & 22\end{array}\right]$} & 11.10 \\
\hline R-mid-occipital & {$\left[\begin{array}{lll}38 & -78 & 28\end{array}\right]$} & 9.52 \\
\hline L-mid-temporal & {$\left[\begin{array}{llll}-50 & -66 & 0\end{array}\right]$} & 7.40 \\
\hline L-inf-frontal & {$\left[\begin{array}{lll}-38 & 18 & 26\end{array}\right]$} & 4.67 \\
\hline
\end{tabular}

BSR $>4 ; p<0.0001$.

active baseline conditions such as the one adopted in the current study (Stark and Squire, 2001). Given that we expected to capture reliable hippocampal activation relevant to episodic memory functions, it was critical to accommodate a suitable baseline task. During each control block (a total of eight blocks), a fixation cross appeared in the center of the visual field for $1-2.5 \mathrm{~s}$ and was briefly replaced with a circle that appeared for $0.5 \mathrm{~s}$. The circle was followed by another fixation cross, which was presented for 2-3.5 s. Participants were instructed to indicate, as quickly as possible, when the fixation cross changed to a circle.

Image acquisition. $\mathrm{fMRI}$ data were collected on a $3 \mathrm{~T}$ Discovery MR750 (General Electric) scanner with a 32-channel head coil. Scanner parameters for the gradient-echo EPI sequence were as follows: TR $=2.0 \mathrm{~s}, 37$ slices ( $3.9 \mathrm{~mm}$ thick), $96 \times 96$ matrix, FOV $=250 \mathrm{~mm}$, flip angle $=80^{\circ}$, $\mathrm{TE}=30 \mathrm{~ms}$. Ten dummy scans were collected to allow for equilibration of the fMRI signal. Stimuli were presented on a computer screen that was seen by participants through a tilted mirror attached to the head coil. E-prime (Psychology Software Tools; www.pstnet.com/eprime) was used for presentation of the stimuli and the responses were made on an MR-compatible response pad (Current Designs). Structural highresolution T1-weighted images ( 180 slices, $1 \mathrm{~mm}$ thickness, FOV $=25 \times$ $25 \mathrm{~cm}$, voxel size $=0.5 \times 0.5 \times 1 \mathrm{~mm}^{3}$ ) were collected following the functional images.

Data analysis. Functional and structural images were preprocessed using statistical parametric mapping software (SPM8; Wellcome Department of Imaging Science, Functional Imaging Laboratory). All functional images were first corrected for acquisition time differences between slices within each volume. The slice-timing corrected images were then rigidly aligned to the first image volume to correct for head movement. A within-subject rigid registration was conducted to align functional and structural images together. T1-weighted images were segmented into gray matter (GM) and white matter (WM) using a new segmentation algorithm implemented in SPM8 [modified version of unified segmentation by (Ashburner and Friston, 2005)]. Then, a groupspecific template was created using Diffeomorphic Anatomical Registration Using Exponentiated Lie Algebra (DARTEL) (Ashburner, 2007). This was done by first importing tissue class images (e.g., GM, WM) into the DARTEL space using the normalization parameter yielded during the segmentation step followed by resampling to isotropic voxels $(1.5 \times$ $1.5 \times 1.5 \mathrm{~mm})$. Then, the imported images went through an iterative 
procedure that began by producing an initial template as a mean of GM/WM across all participants $(n=292)$. Deformation from the initial template to each of the subject-specific GM/WM images was computed and the inverse of the deformation was applied to each of the subject-specific GM/WM images. A second template was then created as the mean of the deformed subject-specific GM/WM images across all participants and this procedure was repeated until a sixth template was created. Finally, the coregistered fMRI images and segmented GM/WM images were nonlinearly normalized, subject by subject, to the sample-specific template (using a subjectspecific flow field), affine aligned into a Montreal Neurological Institute template, and finally smoothed using a $8.0 \mathrm{~mm}$ fullwidth at half-maximum Gaussian filter. Thus, both preprocessed structural MRI and fMRI images were in the same space and had the same voxel size $(1 \times 1 \times 1 \mathrm{~mm})$.

The preprocessed fMRI image data were analyzed with spatiotemporal PLS (McIntosh and Lobaugh, 2004; McIntosh et al., 2004), which determines time-varying distributed patterns of brain activity associated with either some aspects of the experimental design or a behavioral measure. This brain activity encompasses those voxels whose signal change covaries with the experimental conditions/behavioral measure in the same way. PLS assesses activation changes across all regions of the brain simultaneously rather than tessellations of regions, thereby eliminating the need for regional multiple-comparison correction. Additionally, such an analysis accommodates all task conditions into the analyses simultaneously, which facilitates the identification of both common and unique brain activity across conditions. A detailed description of the spatiotemporal analyses for (event-related) fMRI data has been given previously (Salami et al., 2010). In short, a data matrix was created by including each individual's fMRI block onsets and durations for each condition. We also used SPM's hemodynamic response function (HRF) model to create the data matrix. A cross-block covariance matrix between changes in brain activity and experimental conditions was then subjected to mean centering by subtracting the mean of each column from each value of that column. Singular value decomposition (SVD) was conducted on the mean-centered data matrix to reveal orthogonal latent variables (LVs), which reflect cohesive patterns of brain activity related to the experimental conditions. The first and successive LVs account for the greatest and progressively less amount of the cross-covariance matrix, respectively. Within each LV, voxel and design saliencies represent the relation of each voxel and experimental design to the LVs, correspondingly. In addition, brain scores and design scores were computed for each LV. The former was obtained as a dot product of each subject's image volume and voxel saliencies of each LV to derive an estimate of how strong each subject contributes to the pattern of each LV. The latter was computed as a dot product of each subject's image volume and design saliencies of each LV to derive an estimate of how much each particular task is related to weighted voxels of each LV.

In correspondence with the number of tasks, three LVs were extracted from the SVD. To assess the statistical significance of each LV, 500 permutation tests were conducted to determine whether the overall pattern in each LV was significantly different from randomness. The statistical cutoff was set to $p<0.001$. In addition, 100 bootstrap tests were performed to derive an estimate of how reliably each voxel salience contributed to the pattern of LVs. The reliability of each voxel was assessed by the bootstrap ratio (BSR), which is the ratio of the voxel salience to the SE of that salience. Voxels with BSR $>4$, which approximate a $p$ value of 0.0001 , were considered reliable. In addition, bootstrap resampling was used to generate $95 \%$ confidence intervals (CI) around brain scores, which provide a reliable measure to facilitate the interpretation. For example, significant difference between brain scores in different conditions is indicated by nonoverlapping CIs. Similarly, brain scores in a condition were deemed to be unreliable if the CI for that condition crossed zero (McIntosh and Lobaugh, 2004). All the reported clusters consisted of at least 20 contiguous voxels with a minimum distance of $10 \mathrm{~mm}$ between each cluster.

To investigate age-related functional differences during the episodic tasks, a voxelwise general linear model (GLM) was set up for each participant to generate a subject-specific contrast (encoding or retrieval vs baseline). To this end, each condition (encoding, retrieval, and baseline) was modeled as a box-car function that was convolved with the HRF. The six realignment parameters extracted from the realignment algorithm were included in the model as covariates of no interest to account for motion artifacts. Subject-specific contrasts were taken into a second level random-effects model using multiple regressions with age and the square of the orthogonalized age as covariates. Local maxima with $p<0.05$ (familywise error corrected), and an extent threshold of 20 contiguous voxels $(k>20)$ were considered significant for both main and age effects. The age effect was investigated within a mask created from the main effect, thereby reducing the number of comparisons to correct for.

To investigate age-related structural differences, a multiple regression analysis was performed with smoothed normalized GM volumes as a dependent variable and age and the square of orthogonalized age as independent variables. To adjust for intergender variation in brain volume, a subject-specific total intracranial volume was computed by summing the GM and WM segments and was set as a covariate of no interest in the multiple regression analysis.

To explore whether age-related functional differences were driven by local GM atrophy, the Biological Parametric Mapping (BPM) toolbox was used (Casanova et al., 2007). BPM enables multimodal image analyses that model changes in one imaging modality as a function of another modality using the GLM framework. In this context (using GLM), BPM is connatural to SPM, but in addition can use image regressors. Thus, the design matrix in the BPM analysis is voxel specific in contrast to the identical design matrix for all voxels in the traditional SPM analysis. 
Table 3. Correlation between brain score of the entire pattern(from LV2) as well as $\beta$ values of the most contributive regions to episodic networks and age, scanner performance, and measures of the cognitive battery

\begin{tabular}{|c|c|c|c|c|c|c|c|}
\hline Regions & $X Y Z$ & Corr (age, B) & Corr (perf, B) & Corr $(B D, B)$ & Corr (EM, B) & Corr (FL, B) & $\operatorname{Corr}(\mathrm{LD}, \mathrm{B})$ \\
\hline Encoding BS & Overallpattern & $-0.13^{* *}$ & $0.18^{* *}$ & 0.09 & $0.16^{* *}$ & 0.03 & 0.11 \\
\hline \multicolumn{8}{|l|}{ LV2 positive } \\
\hline L-lingual & {$\left[\begin{array}{lll}-16 & -92-12\end{array}\right]$} & $-0.18^{* *}$ & 0.08 & 0.07 & 0.11 & -0.06 & 0.07 \\
\hline L-ant-hippocampus & {$[-28-14-16]$} & $-0.18^{* *}$ & $0.17^{* *}$ & 0.05 & $0.21^{* *}$ & 0.04 & $0.15^{* *}$ \\
\hline L-sup-frontal & {$\left[\begin{array}{llll}-22 & 24 & 56\end{array}\right]$} & -0.09 & 0.10 & 0.08 & $0.14^{*}$ & 0.06 & $0.16^{* *}$ \\
\hline L-mid-frontal & {$\left[\begin{array}{lll}-34 & 20 & 54\end{array}\right]$} & -0.08 & 0.08 & 0.07 & 0.09 & -0.01 & 0.09 \\
\hline L-postcentral & {$\left[\begin{array}{lll}-48 & -14 & 38\end{array}\right]$} & 0.09 & -0.04 & -0.10 & -0.03 & -0.11 & -0.03 \\
\hline R-ant-hippocampus & {$[24-8-18]$} & $-0.19^{* *}$ & $0.17^{* *}$ & 0.03 & $0.19^{* *}$ & 0.04 & $0.16^{* *}$ \\
\hline R-parahippocampus & {$[30-26-12]$} & -0.05 & 0.05 & -0.01 & 0.03 & 0.00 & 0.02 \\
\hline R-pos-hippocampus & {$[24-32-4]$} & $-0.13^{*}$ & 0.03 & 0.00 & 0.06 & -0.09 & 0.02 \\
\hline R-mid-temporal & {$\left[\begin{array}{lll}60 & -60 & 20\end{array}\right]$} & -0.07 & 0.02 & 0.08 & -0.04 & -0.10 & 0.00 \\
\hline L-inf-frontal & {$\left[\begin{array}{lll}-38 & 34 & -14\end{array}\right]$} & -0.01 & 0.11 & -0.04 & $0.15^{* *}$ & 0.00 & 0.06 \\
\hline Retrieval BS & Overall pattern & 0.01 & $0.11^{*}$ & 0.02 & 0.08 & 0.04 & 0.09 \\
\hline \multicolumn{8}{|l|}{ LV2 negative } \\
\hline L-postcentral & {$\left[\begin{array}{lll}-38 & -34 & 44\end{array}\right]$} & -0.06 & -0.02 & 0.03 & -0.05 & 0.01 & -0.06 \\
\hline R-inf-frontal & {$\left[\begin{array}{lll}34 & 26 & -6\end{array}\right]$} & $-0.12^{*}$ & 0.09 & $0.11^{*}$ & 0.07 & 0.00 & 0.10 \\
\hline L-insula & {$\left[\begin{array}{lll}-30 & 24 & -4\end{array}\right]$} & -0.09 & 0.01 & $0.12^{*}$ & 0.07 & 0.06 & 0.10 \\
\hline R-mid-cingulum & {$\left[\begin{array}{lll}8 & 20 & 34\end{array}\right]$} & -0.09 & 0.06 & 0.07 & 0.05 & -0.04 & 0.05 \\
\hline R-cerebellum & {$\left[\begin{array}{lll}22 & -52 & -26\end{array}\right]$} & $-0.12^{*}$ & 0.03 & 0.08 & $0.12^{*}$ & 0.07 & 0.06 \\
\hline L-mid-temporal & {$\left[\begin{array}{lll}-50 & -66 & 0\end{array}\right]$} & 0.04 & -0.03 & 0.04 & -0.06 & 0.00 & -0.04 \\
\hline L-inf-frontal & {$\left[\begin{array}{lll}-38 & 18 & 26\end{array}\right]$} & -0.03 & 0.02 & 0.09 & 0.05 & 0.02 & 0.04 \\
\hline
\end{tabular}

$\mathrm{BD}$, block design; BS, brain score; EM, episodic memory; $\mathrm{FL}$, fluency; LD, letter digit; perf, retrieval performance. ${ }^{*} p<0.05,{ }^{* *} p<0.01$.

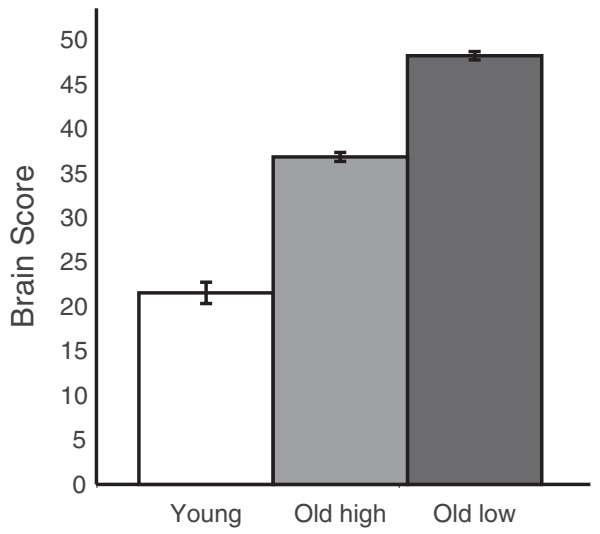

Figure 3. Upregulated recruitment of the task-general network as a function of encodingspecific network integrity. Age- and performance-matched older adults were subdivided on basis of their engagement of the process-specific encoding network (LV2). The bar graph displays the corresponding engagement of the process-general network (LV1). Process-general network engagement, as reflected by brain scores, was highest for older adults with low engagement of the encoding network (old low), intermediate for elderly with higher encodingnetwork recruitment (old high), and lowest for younger adults.

Local maxima with $p<0.05$ (false discovery rate corrected) were considered to be significant.

\section{Results}

\section{Behavioral results}

During the retrieval task, $65.64 \%$ (15.75 \pm 3.13 items $)$ of the faces were correctly retrieved whereas $21.66 \%$ (5.19 \pm 2.82 items) and $12.70 \%$ (3.04 \pm 2.59 items) were associated to incorrect responses and nonresponses, respectively. The number of correctly retrieved names during scanning declined as a function of increasing age $(r=-0.47 ; p<0.0001)$. Also, mean reaction time for correctly retrieved items increased as a function of increasing age $(r=0.49 ; p<0.0001)$.

\section{fMRI results}

Two significant LVs were identified in the task PLS analysis. LV1 $(p<0.0001$; Fig. $1 A)$ accounted for $93.73 \%$ of the cross-block covariance and identified brain regions differentiating both task conditions from baseline, with the largest differentiation between the retrieval and baseline conditions (Fig. $1 B$ ). Peak voxels from regions contributing to the pattern in LV1 are given in Table 1. The signal changes for selected regional maxima are shown in Figure 1, $C$ and $D$. Regions showing greater activity during both tasks compared with baseline included the inferior parietal lobule, the middle and inferior frontal gyrus, the fusiform gyrus, the cerebellum, the posterior hippocampus, the occipital gyrus, the lingual gyrus, and the insula, bilaterally. Brain regions with relatively more activity during baseline included bilateral superior and middle temporal gyrus, bilateral putamen, bilateral superior and medial frontal gyrus, bilateral fusiform gyrus, and right thalamus.

LV2 ( $p<0.0001$; Fig. $1 E$ ) accounted for $6.37 \%$ of the crossblock covariance. This LV identified brain regions whose activity differentiated between the encoding and retrieval conditions, whereas the baseline condition did not reliably contribute to this pattern (CI crossing zero; Fig. $1 F$ ). Table 2 summarizes the significant regional saliencies in LV2. The positive saliencies correspond to greater activity during encoding than during retrieval and included bilateral parietal cortex, bilateral middle frontal gyrus (more extended in the left hemisphere), bilateral anterior hippocampus, bilateral superior occipital gyrus, left middle occipital gyrus, and right fusiform gyrus. The negative saliencies reflected greater activity during retrieval than during encoding and included bilateral cerebellum, right lingual gyrus, left middle occipital gyrus, right precuneus, the right insula, and the right inferior frontal gyrus. The signal change for specific regional maxima is shown in Figure $1, G$ and $H$, and confirms previous reports of asymmetric encoding-retrieval activations in the prefrontal cortex (PFC; Nyberg et al., 1996a; Habib et al., 2003).

The PLS analysis identified distinct encoding and retrieval networks (LV2), and separated these from a process-general network (LV1). Figure 2, $A$ and $C$, illustrates the process-general recruitment of the posterior MTL and the encoding-specific recruitment of anterior hippocampus, respectively (Lepage et al., 1998; Schacter and Wagner, 1999; Persson et al., 2011). It should, 


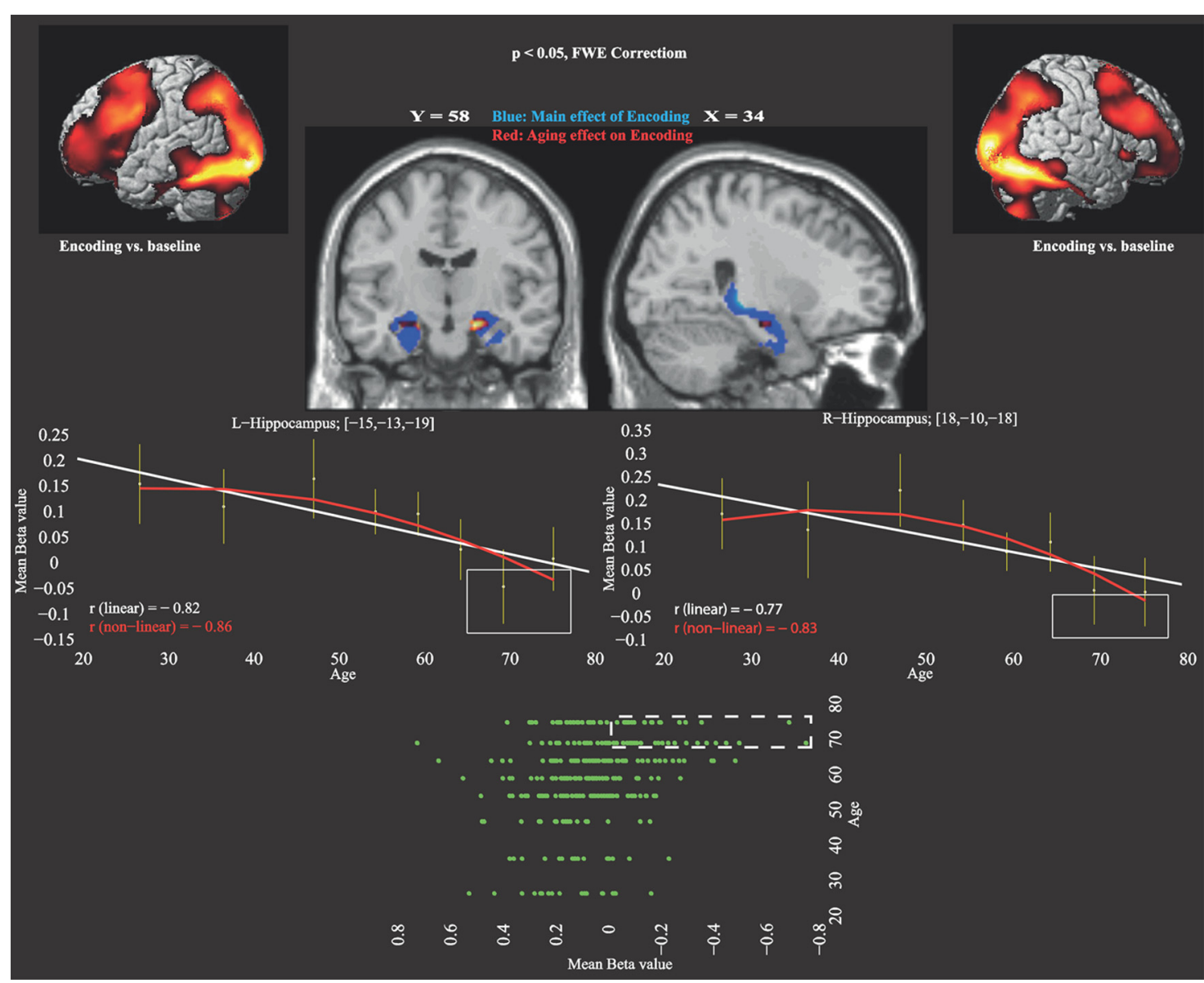

Figure 4. Age effects in relation to brain activity during encoding. The upper part displays brain activity for the main effect of encoding versus baseline (cortical renderings in the top corners). Main (blue) and age (red) effects of encoding in the hippocampus are shown on sagittal and coronal slices. The line graphs show differences in hippocampal activity ( $\beta$ values from the regression analysis) as a function of age. The age-related functional decline in the hippocampus accelerated with increasing age. Rectangles highlight participants in the oldest group (70 - 80 years) who had lowest hippocampal activation. At bottom, a plot of the hippocampal $\beta$-value distribution across all participants $(n=292)$. Dashed rectangle indicates participants with lowest hippocampal activation. $L$, left; r, right.

though, be stressed that the pattern as a whole provides a better relation to the experimental conditions than any individual region. To further characterize the encoding and retrieval networks, individual brain scores were correlated with age, memory performance from the retrieval phase of the FN-PA, and performance on the different measures in the off-line cognitive battery (see Table 3).

LV1 identified a process-general effect, common to both encoding and retrieval, and the brain scores from encoding and retrieval were therefore averaged for the correlation analyses. The brain scores showed a significant positive correlation with age $(r=0.16$; $p<$ $0.01)$ as well as significant negative correlations with episodic memory performance as measured during scanning $(r=-0.21 ; p<0.01)$ and in the off-line battery $(r=-0.16 ; p<0.01)$. Thus, the brain network identified by LV1 was recruited to a greater extent by older adults, and stronger engagement of this network correlated with lower episodic memory performance.

Next, brain scores for encoding and retrieval, identified by LV2, were related to age and cognitive performance. Encoding brain scores were negatively correlated with age $(r=-0.13$; $p<$ 0.01 ), showing that older adults tended to recruit this network to a lesser degree. Also, the encoding brain scores showed a selective positive correlation with episodic memory performance (scanner performance: $r=0.16, p<0.01$; off-line battery: $r=0.18, p<0.01$ ), but not with the performance on nonepisodic tasks (Table 3 ). Thus, stronger engagement of this network during encoding was related to higher levels of episodic memory performance. For retrieval, the correlations were nonsignificant.

The opposing relation between age and recruitment of the process-general (LV1) and process-specific (LV2) networks indicated that an age effect on the hippocampus-based encoding network cascades into upregulated recruitment of the general network. By this view, elderly with more pronounced decline in the episodic encoding network should engage the general network to a particularly high degree. We tested this prediction by identifying a subgroup of elderly ( $>60$ years old, $n=90$; mean age: $64.40 \pm 7.36$ years; mean hit rate: $15.21 \pm 3.05$ items) with lowest engagement of the process-specific encoding network according to a median-split analysis. We then examined whether this group showed higher recruitment of the general network as compared with age- and performance-matched elderly counterparts $(n=91$; mean age: $64.12 \pm 6.69$ years; mean hit rate: $15.04 \pm 2.85$ items) who engaged the process-specific encoding network to a greater degree. A significant difference was observed, confirming that lower process-specific network recruitment predicted higher engagement of the process-general network $\left(t_{(179)}=2.52, p<0.01\right.$; Fig. 3$)$.

The bilateral hippocampus was a salient component of the encoding network. We supplemented the analyses of correlations 
Table 4. Regions showing age-related GM loss with increasing age

\begin{tabular}{|c|c|c|c|}
\hline Regions & $X Y Z$ & $T$ value & Cluster size \\
\hline L-thalamus & {$\left[\begin{array}{lll}-1 & -97\end{array}\right]$} & 11.89 & 34283 \\
\hline L-amygdala & {$\left[\begin{array}{lll}-180 & -15\end{array}\right]$} & 11.09 & Subreg \\
\hline L-hippocampus & {$\left[\begin{array}{lll}-14 & -34 & 3\end{array}\right]$} & 10.28 & Subreg \\
\hline L-sup-temporal & {$[-60-118]$} & 8.50 & Subreg \\
\hline L-putamen & {$\left[\begin{array}{lll}-17 & 11 & -3\end{array}\right]$} & 6.93 & Subreg \\
\hline L-hippocampus & {$[-29-11-10]$} & 6.60 & Subreg \\
\hline L-inf-frontal & {$\left[\begin{array}{llll}-5 & 11 & 19\end{array}\right]$} & 6.06 & Subreg \\
\hline L-mid-temporal & {$\left[\begin{array}{lll}-62 & -51 & 9\end{array}\right]$} & 10.45 & 104995 \\
\hline L-postcentral & {$\left[\begin{array}{lll}-24 & -28 & 72\end{array}\right]$} & 10.13 & Subreg \\
\hline L-ant-cingulum & {$\left[\begin{array}{lll}-1 & 41 & 27\end{array}\right]$} & 10.19 & 32499 \\
\hline L-sup-frontal & {$\left[\begin{array}{llll}-7 & 65 & 5\end{array}\right]$} & 9.53 & Subreg \\
\hline L-mid-frontal & {$\left[\begin{array}{llll}-31 & 60 & 7\end{array}\right]$} & 8.14 & Subreg \\
\hline L-inf-frontal & {$\left[\begin{array}{llll}-47 & 36 & 17\end{array}\right]$} & 7.12 & Subreg \\
\hline R-amygdala & {$\left[\begin{array}{lll}18 & 1 & -13\end{array}\right]$} & 8.85 & 5653 \\
\hline R-hippocampus & {$[17-2-12]$} & 8.17 & Subreg \\
\hline R-putamen & [ [27 9 9] & 5.21 & Subreg \\
\hline R-insula & {$\left[\begin{array}{lll}49 & 17 & -5\end{array}\right]$} & 8.10 & 4357 \\
\hline R-inf-frontal & {$[3736-16]$} & 5.61 & Subreg \\
\hline R-calcarine & {$\left[\begin{array}{lll}8 & -60 & 11\end{array}\right]$} & 7.81 & 2069 \\
\hline R-cerebellum & {$[35-60-30]$} & 7.80 & 30992 \\
\hline L-sup-frontal & {$\left[\begin{array}{lll}-9 & 31 & -23\end{array}\right]$} & 6.75 & 1013 \\
\hline R-mid-frontal & {$\left[\begin{array}{llll}31 & 61 & 4\end{array}\right]$} & 6.51 & 2744 \\
\hline L-fusiform & {$\left[\begin{array}{lll}-22 & -44 & -14\end{array}\right]$} & 5.63 & 164 \\
\hline L-inf-frontal & {$\left[\begin{array}{lll}-37 & 36 & -17\end{array}\right]$} & 5.38 & 108 \\
\hline R-fusiform & {$[23-50-14]$} & 5.29 & 155 \\
\hline R-inf-frontal & {$\left[\begin{array}{llll}50 & 15 & 31\end{array}\right]$} & 5.25 & 213 \\
\hline
\end{tabular}

Subreg, subregion. $p<0.05$ (familywise error corrected), $k>20$.

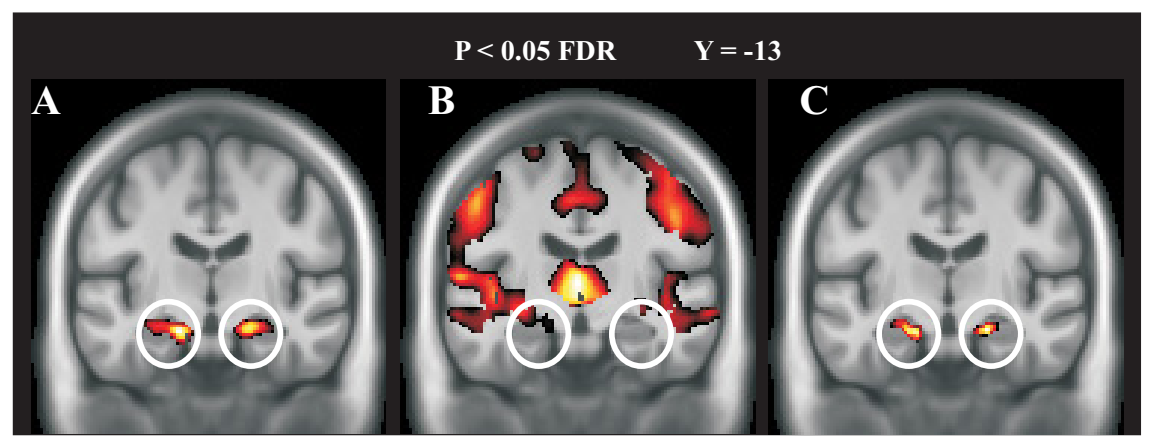

Figure 5. Results from the multimodal BPM analysis overlaid on the sample-specific template. Circles indicate structural and functional age-related decline in bilateral anterior hippocampus. $\boldsymbol{A}$, Regression analysis reflecting age-related functional differences. $\boldsymbol{B}$, Regression analysis demonstrating age-related $\mathrm{GM}$ volume loss. $\boldsymbol{C}$, Regression analysis indicating age-related functional changes after controlling for local GM loss. When controlling for GM loss, age-related functional decline was still apparent in bilateral hippocampus.

between overall brain scores and age/memory performance, with correlations of $\beta$ values (Table 3 ) for bilateral hippocampus. In keeping with the correlation patterns for the overall brain scores, the values for bilateral hippocampus were significantly correlated with age, scanner memory performance, and off-line episodic memory performance. In addition, significant correlations were found for letter digit task performance.

Given the significant correlation between encoding brain scores and age, as well as the correlations of bilateral hippocampal scores with age, a multiple regression analysis across the whole brain was performed to assess age-related functional decline during episodic encoding. The results confirmed a significant association of age and bilateral hippocampus recruitment (left: $x y z=-15-13-19, t_{(290)}=4.59, k=989$; right: $x y z=18-10$ $\left.-18, t_{(290)}=4.56, k=910\right)$ as well as the left lingual gyrus $(x y z=$ $\left.-13-93-13 ; t_{(290)}=4.16 ; k=1043\right)$. Figure 4 illustrates the pattern of age-related functional decline, showing a nonlinear trend, with accelerated hippocampal functional decline in the oldest age groups [left: $r^{2}$ (linear) $=0.67 ; r^{2}$ (nonlinear) $=0.73$; $\Delta r^{2}=6 \%$; right: $r^{2}$ (linear) $=0.59 ; r^{2}$ (nonlinear) $=0.69 ; \Delta r^{2}=$ $10 \%]$. Control analyses showed that the age-related diminished hippocampal activation persisted after controlling for age-related changes in memory performance during scanning (left: $t_{(289)}=$ $3.89, p<0.0001$; right: $\left.t_{(289)}=3.33, p<0.0005\right)$.

The plot of signal changes in the bilateral hippocampus identified some elderly with very low and nonsignificant hippocampal activation (Fig. 4, insert). These "low activators" were screened for ApoE status and Mini Mental State Examination (MMSE) performance (Folstein et al., 1975). Only two were ApoE e4carriers and all scored $>26$ on the MMSE. Moreover, their performance on the tasks in the Betula test battery was within normal levels. Collectively, these results suggest that the low activators were unlikely to be in a preclinical phase of dementia (and all passed clinical screening for dementia before scanning).

\section{Structure-function integration}

The multiple regression analysis of GM volume revealed marked age-related volume reductions in several regions, including the thalamus, amygdala, putamen, hippocampus, the inferior frontal gyrus, and the middle temporal gyrus, bilaterally (Table 4).

In a final set of analyses, functional and structural data were integrated using BPM. Of chief interest was to investigate whether the observation of diminished hippocampal functional responses in aging could be accounted for by age-related local changes in brain GM. The multiple regression approach showed that age-related diminished hippocampal activation persisted after controlling for GM volume, but with smaller extent and a reduced peak value (left: $x y z=-21-16$ $-18, t_{(290)}=3.91, k=815$; right: $x y z=20$ $-10-18, t_{(290)}=3.89, k=585$ ) (Fig. 5).

\section{Discussion}

This study provides novel evidence for opposing effects of age on memory encoding and cognitive control at the systems level. In contrast, most previous studies investigated regional effects of age on memory encoding, retrieval, and cognitive control (i.e., limited evidences at the network level). Using multivariate PLS, we dissociated a process-general (encoding/retrieval) cognitive network from process-specific encoding and retrieval networks. The general network involved large portions of frontoparietal cortex and also cerebellum and posterior hippocampus. Middle and inferior frontal gyrus have been associated with several general cognitivecontrol processes, including top-down attentional control (Banich et al., 2000; Hopfinger et al., 2000; Naghavi and Nyberg, 2005) and attention to targets (Corbetta et al., 2002), as well as response inhibition and selection (Liddle et al., 2001; Corbetta and Shulman, 2002; Aron et al., 2004). Moreover, the inferior and middle frontal gyrus as well as the inferior parietal lobule have been implicated in spatial attention (Naghavi and Nyberg, 2005). The posterior hippocampal activation was common to both conditions, suggesting that this part of the hippocampus is involved in processes that are taxed during both encoding and retrieval. The present face-name task should engage associative operations 
at both processing stages (Nyberg et al., 2000), and previous imaging findings relate associative memory operations to posterior segments of the hippocampus (Henke et al., 1997, 1999; Kirwan and Stark, 2004).

The second LV revealed distinct networks for encoding and retrieval. Differential patterns of prefrontal recruitment contributed to this effect. Consistent with the hemispheric encoding/ retrieval asymmetry (HERA) model (Tulving et al., 1994; Nyberg et al., 1996a; Habib et al., 2003), a left PFC region was involved during episodic encoding (Fig. $1 H$ ) whereas a right PFC region was more involved during episodic retrieval (Fig. $1 G$ ). Notably, in keeping with previous findings [(Schacter and Wagner, 1999; Persson et al., 2011], bilateral anterior hippocampus was differentially more engaged during episodic encoding. This effect might reflect binding processes that are of particular relevance for the formation of new associations in memory (Chua et al., 2007).

Brain scores from the general network and the processspecific networks were related to age and cognitive performance and strikingly different patterns were observed. The processgeneral brain network was recruited to a greater extent by older adults (positive correlation between brain scores and chronological age). Stronger engagement of this network predicted lower performance on episodic memory tasks. In contrast, the encoding network was recruited to a lesser degree by older adults, and greater engagement of this network during encoding was related to higher performance levels for episodic memory task. Moreover, elderly with lesser engagement of the process-specific encoding network recruited the process-general network more than elderly with greater engagement of the process-specific encoding network. Together, these findings suggest that age-related episodic memory impairment may be caused by inefficient use of the encoding network (Naveh-Benjamin et al., 2003), which in turn cascades into a greater reliance on general cognitive-control processes (Gazzaley et al., 2008).

The analyses converged to show that age-related functional changes in the hippocampus contributed to inefficient encoding. Both simple correlational and multiple regression analyses (across the entire brain) confirmed that the strongest age-related functional decline was seen in the anterior hippocampus. This effect remained after controlling for age-related performance differences. It has been debated whether hippocampal structure and function is affected in normal, nonpathological aging (for review, see Buckner, 2004; Hedden and Gabrieli, 2004). Some previous studies reported age-related hippocampus hypoactivation (Gutchess et al., 2005; Daselaar et al., 2006), whereas others found preservation of hippocampus responses across the examined age span (Persson et al., 2011). The present results indicate that age-related reductions in hippocampal functional responses are relatively minor before age 70, suggesting that differences in age ranges across studies may account for some between-studies discrepancies. Elderly who displayed weak hippocampus engagement were scrutinized for ApoE status, MMSE score, and cognitive status. No evidence was obtained to support the view that hippocampal under-recruitment was related to pathological processes such as dementia.

The structural analyses provided evidence for age-related atrophy in several brain regions, including the hippocampus. The observed pattern was broadly consistent with that seen in previous studies (Raz et al., 2005; Fjell et al., 2009). We have previously observed that structural changes in frontal cortex were related to age-related reduction of prefrontal activation during cognitive tasks (Nyberg et al., 2010). Here, when functional and structural data were inter-related, overlap was observed in left an- terior hippocampus, indicating that structural changes in the hippocampus, at least in part, contributed to the age-related under-recruitment of the functional encoding network. However, the age-related structural change did not completely mediate the age-related changes in hippocampus activation (i.e., the effect persisted after controlling for GM volume; Fig. 5), and several other factors might be of relevance, including agerelated molecular changes (Chételat et al., 2011) and changes in the pattern of the hypothalamic-pituitary adrenal axis (Miller and O'Callaghan, 2003).

The PLS analysis of the data from the present large-scale dataset was instrumental for the identification of a general network along with specific episodic memory networks. Importantly, PLS opened up for the use of subject-specific brain scores as indicators of a person's large-scale brain network status. Such integrated scores could be valuable in imaging genetics studies, in particular in efforts to combine imaging and genetic data from genome-wide association studies. A potential limitation is that the findings were derived from a blocked statistical model, as PLS is not a suitable tool for fast event-related designs (McIntosh et al., 1996; McIntosh and Lobaugh, 2004). Also, in the current study, only a subsample of participants fulfilled the criterion of having a sufficient number of items (remembered vs forgotten). However, the event-related control analysis using a multiple regression approach in SPM revealed similar encoding and retrieval networks, as well as a comparable age-related functional decline in the anterior hippocampus. Another, perhaps more serious, limitation is that the present inferences on age-related changes were based on cross-sectional rather than longitudinal data. Previous studies show that cross-sectional estimates of true age changes may be misleading (Rönnlund et al., 2005; Nyberg et al., 2010). However, cross-sectional and longitudinal results tend to converge for older cohorts (Rönnlund et al., 2005), indicating that the present findings of hippocampal functional reduction that primarily was expressed in the oldest cohorts may be valid.

In conclusion, the results of this study support a model where age-related structural brain changes, in the hippocampus and elsewhere, translates into age-related reductions in the efficient recruitment of a process-specific encoding network along with upregulated recruitment of a general cognitive control network. As such, the findings suggest that age-related decline in memory can be linked to encoding processes as well as to general cognitive-control operations.

\section{References}

Aron AR, Robbins TW, Poldrack RA (2004) Inhibition and the right inferior frontal cortex. Trends Cogn Sci 8:170-177.

Ashburner J (2007) A fast diffeomorphic image registration algorithm. Neuroimage 38:95-113.

Ashburner J, Friston KJ (2005) Unified segmentation. Neuroimage 26:839-851.

Banich MT, Milham MP, Atchley R, Cohen NJ, Webb A, Wszalek T, Kramer AF, Liang ZP, Wright A, Shenker J, Magin R (2000) fMRI studies of Stroop tasks reveal unique roles of anterior and posterior brain systems in attentional selection. J Cogn Neurosci 12:988-1000.

Buckner RL (2004) Memory and executive function in aging and AD: multiple factors that cause decline and reserve factors that compensate. Neuron 44:195-208.

Cabeza R, Nyberg L (2000) Imaging cognition II: an empirical review of 275 PET and fMRI Studies. J Cogn Neurosci 12:1-47.

Cabeza R, Grady CL, Nyberg L, McIntosh AR, Tulving E, Kapur S, Jennings JM, Houle S, Craik FI (1997) Age-related differences in neural activity during memory encoding and retrieval: a positron emission tomography study. J Neurosci 17:391-400.

Casanova R, Srikanth R, Baer A, Laurienti PJ, Burdette JH, Hayasaka S, Flowers L, Wood F, Maldjian JA (2007) Biological parametric mapping: A 
statistical toolbox for multimodality brain image analysis. Neuroimage 34:137-143.

Chételat G, Villemagne VL, Pike KE, Ellis KA, Bourgeat P, Jones G, O’Keefe GJ, Salvado O, Szoeke C, Martins RN, Ames D, Masters CL, Rowe CC (2011) Independent contribution of temporal beta-amyloid deposition to memory decline in the pre-dementia phase of Alzheimer's disease. Brain 134:798-807.

Chua EF, Schacter DL, Rand-Giovannetti E, Sperling RA (2007) Evidence for a specific role of the anterior hippocampal region in successful associative encoding. Hippocampus 17:1071-1080.

Corbetta M, Shulman GL (2002) Control of goal-directed and stimulusdriven attention in the brain. Nat Rev Neurosci 3:201-215.

Corbetta M, Kincade JM, Shulman GL (2002) Neural systems for visual orienting and their relationships to spatial working memory. J Cogn Neurosci 14:508-523.

Daselaar SM, Fleck MS, Dobbins IG, Madden DJ, Cabeza R (2006) Effects of healthy aging on hippocampal and rhinal memory functions: an eventrelated fMRI study. Cereb Cortex 16:1771-1782.

Fjell AM, Walhovd KB, Fennema-Notestine C, McEvoy LK, Hagler DJ, Holland D, Brewer JB, Dale AM (2009) One-year brain atrophy evident in healthy aging. J Neurosci 29:15223-15231.

Folstein MF, Folstein SE, McHugh PR (1975) Mini-Mental State Examination (MMSE). A practical method for grading the cognitive state of patients for the clinician. J Psychiatr Res 12:189-198.

Foster JK, Jelicic M (1999) Memory: systems, process, or function? Debates in psychology. New York: Oxford UP.

Gabrieli JD, Brewer JB, Desmond JE, Glover GH (1997) Separate neural bases of two fundamental memory processes in the human medial temporal lobe. Science 276:264-266.

Gazzaley A, Clapp W, Kelley J, McEvoy K, Knight RT, D’Esposito M (2008) Age-related top-down suppression deficit in the early stages of cortical visual memory processing. Proc Natl Acad Sci U S A 105:13122-13126.

Giovanello KS, Schnyer D, Verfaellie M (2009) Distinct hippocampal regions make unique contributions to relational memory. Hippocampus 19:111-117.

Grady CL, McIntosh AR, Horwitz B, Maisog JM, Ungerleider LG, Mentis MJ, Pietrini P, Schapiro MB, Haxby JV (1995) Age-related reductions in human recognition memory due to impaired encoding. Science 269: $218-221$.

Grady CL, Springer MV, Hongwanishkul D, McIntosh AR, Winocur G (2006) Age-related changes in brain activity across the adult lifespan. J Cogn Neurosci 18:227-241.

Greicius MD, Krasnow B, Boyett-Anderson JM, Eliez S, Schatzberg AF, Reiss AL, Menon V (2003) Regional analysis of hippocampal activation during memory encoding and retrieval: fMRI study. Hippocampus 13:164-174.

Gutchess AH, Welsh RC, Hedden T, Bangert A, Minear M, Liu LL, Park DC (2005) Aging and the neural correlates of successful picture encoding: frontal activations compensate for decreased medial-temporal activity. J Cogn Neurosci 17:84-96.

Habib R, Nyberg L, Tulving E (2003) Hemispheric asymmetries of memory: the HERA model revisited. Trends Cogn Sci 7:241-245.

Hedden T, Gabrieli JD (2004) Insights into the ageing mind: a view from cognitive neuroscience. Nat Rev Neurosci 5:87-96.

Henke K, Buck A, Weber B, Wieser HG (1997) Human hippocampus establishes associations in memory. Hippocampus 7:249-256.

Henke K, Weber B, Kneifel S, Wieser HG, Buck A (1999) Human hippocampus associates information in memory. Proc Natl Acad Sci U S A 96:5884-5889.

Hopfinger JB, Buonocore MH, Mangun GR (2000) The neural mechanisms of topdown attentional control. Nat Neurosci 3:284-291.

Kirwan CB, Stark CE (2004) Medial temporal lobe activation during encoding and retrieval of novel face-name pairs. Hippocampus 14:919-930.

Lepage M, Habib R, Tulving E (1998) Hippocampal PET activations of memory encoding and retrieval: the HIPER model. Hippocampus 8:313-322.

Liddle PF, Kiehl KA, Smith AM (2001) An event-related fMRI study of response inhibition. Hum Brain Mapp 12:100-109.

McIntosh AR, Lobaugh NJ (2004) Partial least squares analysis of neuroimaging data: applications and advances. Neuroimage 23 [Suppl 1]:S250-S263.

McIntosh AR, Bookstein FL, Haxby JV, Grady CL (1996) Spatial pattern analysis of functional brain images using partial least squares. Neuroimage 3:143-157.
McIntosh AR, Chau WK, Protzner AB (2004) Spatiotemporal analysis of event-related fMRI data using partial least squares. Neuroimage 23:764-775.

Miller DB, O'Callaghan JP (2003) Effects of aging and stress on hippocampal structure and function. Metabolism 52:17-21.

Naghavi HR, Nyberg L (2005) Common fronto-parietal activity in attention, memory, and consciousness: shared demands on integration? Conscious Cogn 14:390-425.

Naveh-Benjamin M, Hussain Z, Guez J, Bar-On M (2003) Adult age differences in episodic memory: further support for an associative-deficit hypothesis. J Exp Psychol Learn Mem Cogn 29:826-837.

Nilsson L-G, Bäckman L, Erngrund K, Nyberg L, Adolfsson R, Bucht G, Karlsson S, Widing M, Winblad B (1997) The betula prospective cohort study: memory, health, and aging. Aging Neuropsychol Cogn 4:1-32.

Nilsson L-G, Adolfsson R, Bäckman L, de Frias C, Molander B, Nyberg L (2004) Betula: a prospective cohort study on memory, health and aging. Aging Neuropsychol Cogn 11:134-148.

Nyberg L, Cabeza R, Tulving E (1996a) PET studies of encoding and retrieval: the HERA model. Psychonomic Bull Rev 3:135-148.

Nyberg L, Bäckman L, Erngrund K, Olofsson U, Nilsson L-G (1996b) Age differences in episodic memory, semantic memory, and priming: relationships to demographic, intellectual, and biological factors. J Gerontol B Psychol Sci Soc Sci 51:234-240.

Nyberg L, McIntosh AR, Cabeza R, Habib R, Houle S, Tulving E (1996c) General and specific brain regions involved in encoding and retrieval of events: What, where, and when. Proc Natl Acad Sci U S A 93:11280-11285.

Nyberg L, Persson J, Habib R, Tulving E, McIntosh AR, Cabeza R, Houle S (2000) Large scale neurocognitive networks underlying episodic memory. J Cogn Neurosci 12:163-173.

Nyberg L, Sandblom J, Jones S, Neely AS, Petersson KM, Ingvar M, Bäckman L (2003) Neural correlates of training-related memory improvement in adulthood and aging. Proc Natl Acad Sci U S A 100:13728-13733.

Nyberg L, Salami A, Andersson M, Eriksson J, Kalpouzos G, Kauppi K, Lind J, Pudas S, Persson J, Nilsson LG (2010) Longitudinal evidence for diminished frontal cortex function in aging. Proc Natl Acad Sci U S A 107:22682-22686.

Persson J, Kalpouzos G, Nilsson LG, Ryberg M, Nyberg L (2011) Preserved hippocampus activation in normal aging as revealed by fMRI. Hippocampus 7:753-766.

Raz N, Lindenberger U, Rodrigue KM, Kennedy KM, Head D, Williamson A, Dahle C, Gerstorf D, Acker JD (2005) Regional brain changes in aging healthy adults: general trends, individual differences and modifiers. Cereb Cortex 15:1676-1689.

Rombouts SA, Barkhof F, Witter MP, Machielsen WC, Scheltens P (2001) Anterior medial temporal lobe activation during attempted retrieval of encoded visuospatial scenes: an event-related fMRI study. Neuroimage 14:67-76.

Rönnlund M, Nyberg L, Bäckman L, Nilsson LG (2005) Stability, growth, and decline in adult life span development of declarative memory: crosssectional and longitudinal data from a population-based study. Psychol Aging 20:3-18.

Salami A, Eriksson J, Kompus K, Habib R, Kauppi K, Nyberg L (2010) Characterizing the neural correlates of modality-specific and modality-independent accessibility and availability signals in memory using partial-least squares. Neuroimage 52:686-698.

Salami A, Eriksson J, Nilsson LG, Nyberg L (2012) Age-related white matter microstructural differences partly mediate age-related decline in processing speed but not cognition. Biochim Biophys Acta 1822:408-415.

Schacter DL, Wagner AD (1999) Medial temporal lobe activations in fMRI and PET studies of episodic encoding and retrieval. Hippocampus 9:7-24.

Sperling RA, Bates JF, Cocchiarella AJ, Schacter DL, Rosen BR, Albert MS (2001) Encoding novel face-name associations: a functional MRI Study. Hum Brain Mapp 14:129-139.

Stark CE, Squire LR (2001) When zero is not zero: the problem of ambiguous baseline conditions in fMRI. Proc Natl Acad Sci U S A 98:12760-12766.

Tulving E (1983) Elements of episodic memory. Oxford: Clarendon.

Tulving E, Kapur S, Craik FI, Moscovitch M, Houle S (1994) Hemispheric encoding/retrieval asymmetry in episodic memory: positron emission tomography findings. Proc Natl Acad Sci U S A 91:2016-2020.

Zeineh MM, Engel SA, Thompson PM, Bookheimer SY (2003) Dynamics of the hippocampus during encoding and retrieval of face-name pairs. Science 299:577-580. 